\title{
Monitoring children after hypospadias repair: What is the best plan of action?
}

\author{
Armando J. Lorenzo, MD, MSc, FRCSC, FAAP, FACS
}

Division of Pediatric Urology, Hospital for Sick Children, University of Toronto, Toronto, ON

See related article on page 238 .

Cite as: Can Urol Assoc J 2012;6(4):243-4. http://dx.doi.org/10.5489/cuaj.12224

$\mathrm{T}$ he tubularized incised plate (TIP) urethroplasty has been carefully scrutinized by many, rightfully maintaining its position as the number one technique for most defects, particularly distal ones. ${ }^{1}$ Nevertheless, while our experience (and the age of patients who have undergone this repair) matures, there continues to be questions related to the quality of the neourethra in terms of diameter and elasticity. Thankfully, most surgeons have now departed from the questionable practice of routine regular urethral "calibration" after surgery, ${ }^{2}$ favouring non-invasive assessment of toilet-trained children by means of a urinary flow rate and ultrasound determination of post-void residuals. For better or worse, we are then faced with seemingly "asymptomatic" young boys with parameters that are either considered abnormal or fall outside of nomogram curves adjusted for age. These findings can somewhat temper the enthusiastic embrace of the TIP technique due to the possibility of longterm consequences from distal urinary flow impairment. Is this a founded source of worry or an unnecessary concern?

There are important issues that come up when reviewing the literature and monitoring children in everyday practice. Aside from questions regarding what a "normal" flow rate in young (recently toilet trained) children represents, and the effect of age and the underlying condition on flow dynamics, ${ }^{3}$ there are some specific aspects of care I find particularly challenging. First, I struggle with being able to confidently say that a young patient is asymptomatic at an age when that assessment is difficult at best. Kids who grow up with a functional urinary flow abnormality would not necessarily perceive it as an impediment and would likely be considered "asymptomatic." Perhaps the same postoperative voiding pattern in a young adult would not be labelled as such. I also have a hard time envisioning the "need" for an intervention or why an intervention is "required." Admittedly some children have significant problems (such as high post-void residuals and recurrent infections), yet most do not. As we dig deeper into

the rationale for intervention, it is difficult to quantify the factors that emerge (such as "parental preference" and "surgeon opinion"). Nevertheless, this commonly accepted exclusion criterion eliminates patients who may be identical to those who were monitored, yet not subjected to further surgery. In a retrospective uncontrolled fashion it is hard to envision what dictates taking such a step. Lastly, I question how much of the detected improvement over time is the result of normal development, tissue remodeling or just adaptation to a relative obstruction. This is rather difficult to quantify and may be less important in distal hypospadias, yet probably gains significance as the defects become more severe, the neourethra segment longer, the intraoperative dissection more extensive, and reliance on poorly developed plates more frequent. Unfortunately, it is this latter group that is most worrisome and for which we only have modest amounts of long-term data.

Considering the data presented by Eassa and colleagues, ${ }^{4}$ some could argue that there is little value in obtaining flow rates in asymptomatic children, considering that we either rejoice when we see a normal one, yet are advised to exercise patience and do very little when abnormal. Nevertheless, if a flow rate is obtained, we must then decide on when an abnormality is really something to worry about or act upon. At which point can we rest assured that persistent abnormal findings are likely inconsequential? How will the underlying characteristics of the neourethra (leading to these voiding dynamics) stand the dramatic developmental and functional demands imposed by puberty? Although it is reassuring that many patients improve and do well during short- to mediumterm follow-up, some (perhaps very few) do not. Is there a way to tell them apart? Now that we have some reassurance we need selectivity; we need to be able to separate patients who benefit from close monitoring from those who can be safely discharged from regular assessment. Experience with "excellent" results that led to not-so-excellent long-term outcomes $^{5,6}$ should encourage us to consider prospectively capturing urinary flow dynamics data and satisfaction of hypospadias patients until early adulthood. 
While we gather more evidence, should we be relieved to see data supporting conservative management and a favourable pattern towards improvement? Yes. Can we apply these findings to all hypospadias patients, including proximal ones? Probably not. Will I continue to regularly monitor patients with an abnormal flow rate after hypospadias repair? You bet!

Competing interests: None declared.

\section{References}

1. Snodgrass WT, Bush N, Cost N. Tubularized incised plate hypospadias repair for distal hypospadias. J Pediatr Urol 2010;6:408-13. http://dx.doi.org/10.1016/i.jpurol.2009.09.010
2. Lorenzo AJ, Snodgrass WT. Regular dilatation is unnecessary after tubularized incised-plate hypospadias repair. BJU Int 2002;89:94-7. http://dx.doi.org/10.1046/j.1464-410X.2002.02519.x

3. Wolffenbuttel KP, Wondergem N, Hoefnagels JJS, et al. Abnormal urine flow in boys with distal hypospadias before and after correction. J Urol 2006;176(4 Pt 2):1733-6, discussion 1736-7.

4. Eassa W, Brzezinski A, Capolicchio JP, et al. How do asymptomatic toilet-trained children void following tubularized incised-plate hypospadias repair? Can Urol Assoc J 2012;6:238-42; http://dx.doi. org/10.5489/cuaj.12029

5. Bracka A. Buccal mucosa: good but not perfect. J Urol 2011;185:777-8. http://dx.doi.org/10.1016/j. juro.2010.12.015

6. Bracka A. Hypospadias repair: the two-stage alternative. Br J Urol 1995;76 (Suppl 3):31-41.

Correspondence: Dr. Armando J. Lorenzo, Hospital for Sick Children, University of Toronto, 555 University Ave, Toronto, 0N M5G 1X8; armando.lorenzo@sickkids.ca

\section{CORRECTIONS}

\section{CUA Annual Meeting Abstracts addition}

Cite as: Can Urol Assoc J 2012;6(4):244. http://dx.doi.org/10.5489/cuaj.12208

The following abstract was omitted from the CUA Abstracts Books for 2012.

\section{Do urologists understand how urinary catheters work, and do we use them correctly? Results of a U.S. nationwide survey}

Introduction and objectives: Foley catheters are assumed to drain the bladder to completion. We have previously shown that dependent loops along the drainage tubing create air-locks, which obstruct antegrade urine flow and result in un-drained residual bladder urine. We hypothesized that drainage characteristics of Foley catheters remain poorly understood by urologists and general surgeons. We conducted a nationwide survey of general surgery and urology training program faculty and residents, to assess perceptions of Foley catheter drainage. We designed a novel catheter drainage tube/bag that eliminates air-locks.

Methods: An anonymous illustrated questionnaire assessing Foley catheter use patterns and perception was sent to general surgery and urology residency programs $(\mathrm{N}=108)$ nationwide. A modified catheter drainage tube/bag unit was designed and tested. An ex vivo catheterized bladder model was designed to measure and compare urine drainage rates with the standard drainage system, versus with our novel design.

Results: A total of 307 responses were collected from residents $(55 \%)$ and faculty $(45 \%)$; responses were similar among both groups $(p<0.05)$. The majority reported that at their centers Foley catheter drainage tubes are generally positioned with a dependent loop $(94.1 \%)$, and, that positioning with a dependent loop, versus without $(78.1 \%)$ promoted optimal drainage. Antegrade drainage does not occur with a traditional drainage system when a $>5.5$ inch dependent loop in place. With our proposed design, which eliminates dependent loops, the bladder model emptied to completion consistently.

Conclusions: Traditional Foley catheter drainage systems, as commonly used, evacuate the bladder sub- optimally. More reliable and complete bladder drainage may decrease the incidence of catheter related UTI. The novel modified Foley catheter drainage tube/bag design presented here eliminates dependent loops, to optimize antegrade drainage.

\section{CUA Guideline on incidentally discovered adrenal mass}

Cite as: Can Urol Assoc J 2012;6(4):244. htrp://dx.doi.org/10.5489/cuaj.12209

In the August 2011 issue of CUAJ, there was an error in the algorithm in the CUA Guideline on the management of incidentally discovered adrenal mass.

The algorithm ${ }^{1}$ in the management of enhanced CT with washouts for benign and suspicious phenotypes should be APW/RPW $\geq 60 / 40 \%$ and APW/RPW $\leq 60 / 40 \%$, respectively.

\section{Reference}

1. Kapoor A, Morris T, Rebello R. Guidelines for the management of the incidentally discovered adrenal mass. Can Urol Assoc J 2011;5:241-7. http://dx.doi.org/10.5489/cuaj.11135 